\title{
REGULAR NEIGHBORHOODS ARE NOT TOPOLOGICALLY INVARIANT
}

\author{
BY MITSUYOSHI KATO
}

Communicated by W. Browder, May 1, 1968

In [5], Siebenmann and Sondow have shown that there exist topologically equivalent $\mathrm{PL}(n+3, n+1)$-sphere pairs for $n \geqq 2$ that are combinatorially distinct. In this note, combining their analysis of strong $h$-cobordisms of certain higher dimensional knots and isoneighboring theorem due to Noguchi [3] and [4], we show the following:

ThEOREM. Assume $n=e v e n \geqq 2$. Then there exist infinitely many combinatorially distinct $\mathrm{PL}(n+3, n+1)$-manifold pairs $\left(V_{k}, K_{k}\right)$, $k=1,2, \cdots$, that are not abstract regular neighborhoods but topologically equivalent to an abstract regular neighborhood $\left(V_{0}, K_{0}\right)$.

REMARK. Each submanifold $K_{k}$ is a $\mathrm{PL}(n+1)$-sphere which is 1-flat in $V_{k}$ with only one singularity. (For 1-flat embeddings and singularities, see [3].)

An implication of the Theorem is that regular neighborhoods are not topologically invariant. More explicitly we may say:

COROLlaRy. The collapsing is not topologically invariant.

We note here that $\left(V_{k}, K_{k}\right)$ and $\left(V_{0}, K_{0}\right)$ have the vanishing Whitehead torsion, since $K_{0}$ is simply connected. However, in the subsequent paper [2], we shall show that the topological invariance of Whitehead torsions is equivalent to that of regular neighborhoods of polyhedra in the sufficiently high-dimensional euclidean space.

1. The construction. In the following, we shall use the notations in [5]. However, we shall be concerned mainly with the combinatorial (or PL) objects. By a PL $n$-knot we shall mean a PL $(n+2, n)$ sphere pair $\left(S^{n+2}, L^{n}\right)$ such that $L^{n}$ has a collar neighborhood $\left(L^{n} \times D^{2}\right)$ in $S^{n+2}$ [1] and [4].

Lemma 1. Assume $n=e v e n \geqq 2$. Then there exist infinitely many invertible strong h-cobordisms of PL n-knots

$c_{k}=\left(\left(W_{k}, M_{k}\right) ;\left(S_{0}, L_{0}\right),\left(S_{k}, L_{k}\right)\right), k=1,2, \cdots$, such that

(1) $\left(S_{k}, L_{k}\right)$ and $\left(S_{0}, L_{0}\right)$ are combinatorially equivalent,

(2) $\pi_{1}\left(S_{0}-L_{0}\right) \cong J \times G$, where $J$ and $G$ are the infinite cyclic group and the binary icosahedral group, respectively, and 
(3) $\tau\left(c_{k}\right)=2 k \tau$ for $k=1,2, \cdots$, where $\tau$ is an element of $\mathrm{Wh}(J \times G)$ of infinite order such that when $k \neq j$, there exists no automorphism $\theta$ of $\pi_{1}\left(S_{0}-L_{0}\right)$ making $\theta * \tau\left(c_{k}\right)=\tau\left(c_{j}\right)$.

The proof of Lemma 1 is essentially given in [5]. In particular, it is to be noted that the argument in Construction 2.5 of [5] is valid for $n=$ even $\geqq 2$.

In the subsequent, we shall employ the notations in Lemma 1 and assume $n=$ even $\geqq 2$. First, we form a PL $(n+3, n+1)$-manifold pair $\left(V_{0}, K_{0}\right)=a *\left(S_{0}, L_{0}\right) \cup_{f_{0}}\left(D^{n+1} \times D^{2}, D^{n+1} \times(0)\right)$ from the cone ball pair $a *\left(S_{0}, L_{0}\right)\left(=\left(a * S_{0}, a * L_{0}\right)\right)$ by attaching the standard ball pair $\left(D^{n+1} \times D^{2}, D^{n+1} \times(0)\right)$ by a PL homeomorphism

$$
f_{0}:\left(b D^{n+1} \times D^{2}, b D^{n+1} \times(0)\right) \rightarrow\left(\left(L_{0} \times D^{2}\right), L_{0}\right) \subset\left(S_{0}, L_{0}\right),
$$

where $b D^{n+1}$ stands for the boundary of the $(n+1)$-ball $D^{n+1}$. Then $\left(V_{0}, K_{0}\right)$ is clearly an abstract regular neighborhood and $K_{0}$ is 1 -flat in $V_{0}$ with only one singularity $\left(S_{0}, L_{0}\right)$ at $a$.

In the same way, we have a $\operatorname{PL}(n+3, n+1)$-manifold pair $\left(V_{k}, K_{k}\right)$ $=a *\left(S_{0}, L_{0}\right) \cup\left(W_{k}, M_{k}\right) \cup_{f_{k}}\left(D^{n+1} \times D^{2}, D^{n+1} \times(0)\right)$ for each $k \geqq 1$, where $f_{k}:\left(b D^{n+1} \times D^{2}, b D^{n+1} \times(0)\right) \rightarrow\left(\left(L_{k} \times D^{2}\right), L_{k}\right) \subset\left(S_{k}, L_{k}\right)$ is a PL homeomorphism.

2. Distinguishing the pairs combinatorially. Let $U_{k}$ be a regular neighborhood of $K_{k}$ in $V_{k}$ such that $U_{k} \subset$ Int $V_{k}$ for each $k \geqq 0$. Since $K_{k}$ is a 1-flat $(n+1)$-sphere in $U_{k}$ with only one singularity $\left(S_{0}, L_{0}\right)$ at $a$ for each $k \geqq 0$, it follows from the Theorem in [4] that $\left(U_{k}, K_{k}\right)$ is combinatorially equivalent to $\left(V_{0}, K_{0}\right)$. Thus we proved:

AsSERTION 1. For each $k \geqq 0$, the abstract regular neighborhoods $\left(U_{k}, K_{k}\right)$ and $\left(V_{0}, K_{0}\right)$ are combinatorially equivalent.

Putting $N_{k}=V_{k}$-Int $U_{k}$, we examine a PL manifold triad $\left(N_{k} ; b U_{k}, b V_{k}\right)$. From Assertion 1, we may identify $\left(U_{k}, K_{k}\right)$ with $\left(V_{0}, K_{0}\right)$. Note that $b V_{0}=E_{0} \cup \cup^{\prime} D^{n+1} \times b D^{2}$ and hence that $\pi_{1}\left(E_{0}\right) \cong \pi_{1}\left(b V_{0}\right)$, where $E_{0}=S_{0}-\left(L_{0} \times \operatorname{Int} D^{2}\right)$ and $f_{0}^{\prime}=f_{0} \mid b D^{n+1} \times b D^{2}$.

Observe that from the construction of the $h$-cobordism $c_{k}, V_{k}$ is obtained from $V_{0}$ by attaching an $h$-cobordism from $b V_{0}$ with the torsion $2 k \tau$. Here we identify $\operatorname{Wh}\left(\pi_{1}\left(b V_{0}\right)\right)$ with $\operatorname{Wh}\left(\pi_{1}\left(E_{0}\right)\right)$ by the isomorphism $\pi_{1}\left(E_{0}\right) \cong \pi_{1}\left(b V_{0}\right)$. In particular, by the regular neighborhood annulus theorem $\left(N_{0} ; b U_{0}, b V_{0}\right)$ is a product cobordism. Therefore, we may conclude the following:

ASSERTION 2. For each $k \geqq 0$, the triad $\left(N_{k} ; b U_{k}, b V_{k}\right)$ is a PL $h$-cobordism with $\tau\left(N_{k}, b U_{k}\right)=2 k \tau$, where the torsion is identified by the isomorphism $\pi_{1}\left(E_{0}\right) \cong \pi_{1}\left(b V_{0}\right) \cong \pi_{1}\left(b U_{0}\right)$. 
Now we are ready to prove the following:

Proposition 1. The manifold pairs $\left(V_{k}, K_{k}\right), k=1,2, \cdots$, are not abstract regular neighborhoods. If $k \neq j$, then $\left(V_{k}, K_{k}\right)$ and $\left(V_{j}, K_{j}\right)$ are combinatorially distinct for $k \geqq 0$ and $j \geqq 0$.

Proof. First, suppose that $\left(V_{k}, K_{k}\right)$ is an abstract regular neighborhood for some $k \geqq 1$. Then it follows that by the uniqueness of regular neighborhoods $\left(V_{k}, K_{k}\right)$ and $\left(U_{k}, K_{k}\right)$ are combinatorially equivalent. Hence, from Assertion $1,\left(V_{k}, K_{k}\right)$ and $\left(V_{0}, K_{0}\right)$ are combinatorially equivalent. Thus, in order to prove the first statement of Proposition 1 , it suffices to show the second statement. To do this, suppose that there exists a PL homeomorphism $g:\left(V_{k}, K_{k}\right) \rightarrow\left(V_{j}, K_{j}\right)$ for some $k \geqq 0$ and $j \geqq 0$ such that $k \neq j$. Then by the uniqueness of regular neighborhoods we may assume that $g\left(U_{k}\right)=U_{j}$ and hence that $g\left(N_{k}\right)=N_{j}$. Thus the $h$-cobordisms $\left(N_{k} ; b U_{k}, b V_{k}\right)$ and $\left(N_{j} ; b U_{j}, b V_{j}\right)$ are combinatorially equivalent. It follows from the combinatorial invariance theorem of Whitehead torsions that $g_{*}^{\prime}(2 k \tau)=2 j \tau$, where $g^{\prime}=g \mid b U_{k}: b U_{k} \rightarrow b U_{j}$. This contradicts Lemma 1, (3), completing the proof.

3. Finding homeomorphisms. Form $\left(W_{k}^{\prime}, M_{k}^{\prime}\right)$ from $\left(W_{k}, M_{k}\right)$ by attaching a collar $\left(S_{0}, L_{0}\right) \times[0,1)$ naturally at the left end $\left(S_{0}, L_{0}\right)$. Then, from the invertibility of the knot cobordisms we may prove the following by the infinite repetition argument:

Lemma 2. For any $k \geqq 1,\left(W_{k}^{\prime}, M_{k}^{\prime}\right)$ is PL homeomorphic to $\left(S_{k}, L_{k}\right) \times[0,1)$. (For the proof, see Lemma 3.1 in [5].)

From Lemma 2 and the cone extension argument, we have:

Corollary to Lemma 2. Any homeomorphism $h:\left(S_{k}, L_{k}\right) \rightarrow\left(S_{0}, L_{0}\right)$ between the boundaries of $a *\left(S_{0}, L_{0}\right) \cup\left(W_{k}, M_{k}\right)$ and $a *\left(S_{0}, L_{0}\right)$ is extendable to a homeomorphism $g: a *\left(S_{0}, L_{0}\right) \cup\left(W_{k}, M_{k}\right) \rightarrow a *\left(S_{0}, L_{0}\right)$.

Proposition 2. For any $k \geqq 1$, there is a homeomorphism $H:\left(V_{k}, K_{k}\right) \rightarrow\left(V_{0}, K_{0}\right)$.

Proof. Let $h:\left(S_{k}, L_{k}\right) \rightarrow\left(S_{0}, L_{0}\right)$ be a PL homeomorphism between the boundaries of $a *\left(S_{0}, L_{0}\right) \cup\left(W_{k}, M_{k}\right)$ and $a *\left(S_{0}, L_{0}\right)$. Then from the uniqueness of regular neighborhoods we may assume that

$$
h f_{k}\left(b D^{n+1} \times D^{2}, b D^{n+1} \times(0)\right)=f_{0}\left(b D^{n+1} \times D^{2}, b D^{n+1} \times(0)\right) .
$$

It follows from Theorem $\mathrm{C}$ in [1] that

$$
f_{0}^{-1} h f_{k}:\left(b D^{n+1} \times D^{2}, b D^{n+1} \times(0)\right) \rightarrow\left(b D^{n+1} \times D^{2}, b D^{n+1} \times(0)\right)
$$


is extendable to a PL homeomorphism

$$
g:\left(D^{n+1} \times D^{2}, D^{n+1} \times(0)\right) \rightarrow\left(D^{n+1} \times D^{2}, D^{n+1} \times(0)\right) .
$$

Combining this fact and the Corollary to Lemma 2, we conclude that the PL homeomorphism $h:\left(S_{k}, L_{k}\right) \rightarrow\left(S_{0}, L_{0}\right)$ is extendable to the required homeomorphism $H:\left(V_{k}, K_{k}\right) \rightarrow\left(V_{0}, K_{0}\right)$, completing the proof.

Now Propositions 1 and 2 complete the proof of Theorem.

\section{REFERENCES}

1. M. Kato, $A$ concordance classification of PL homeomorphisms of $S^{p} \times S^{q}$, Topology (to appear).

2. - Geometrical operations of Whitehead groups (to appear).

3. H. Noguchi, 1-flat 3-manifolds in 5-space. Osaka J. Math. 1 (1964), 117-125.

4. - One-flat submanifolds with codimension two, Illinois J. Math. (to appear).

5. L. Siebenmann and J. Sondow, Some homeomorphic sphere pairs that are combinatorially distinct, Comment. Math. Helv. 41 (1966-67), 261-272.

Tokyo Metropolitan University, Tokyo, Japan 